\title{
RETRACTED: Dependence of emotional burnout on personality typology in the COVID-19 pandemic
}

\author{
Marina Seluch ${ }^{\mathrm{a}, *}$, Mikhail Volchansky $^{\mathrm{b}}$ and Roman Safronov ${ }^{\mathrm{c}}$ \\ ${ }^{a}$ Amur State University, Blagoveshchensk, Russian Federation \\ ${ }^{\mathrm{b}}$ Volgograd State Medical University, Volgograd, Russian Federation \\ ${ }^{c}$ I.M. Sechenov First Moscow State Medical University (Sechenov University), Moscow, Russian Federation
}

Received 10 March 2021

Accepted 3 May 2021

This article has been retracted, and the online PDF has been watermarked "RETRACTED". The retraction notice is available at https://doi.org/10.3233/WOR-223650.

\section{Introduction}

The current era is characterized by rapid scientific and technological progress, which opens a lot of prospects and opportunities to people, not available before [1]. However, such rapid development of

*Address for correspondence: Marina Seluch, Amur State University, Soviet str., 2, Blagoveshchensk, 675028, Russian Federation. E-mail: m.seluch@ rambler.ru. nearly all spheres of human activity is quite challenging for each individual as it creates tough competition in the labour market. Furthermore, the very attitude to work changes, the work requires an employee to be extremely flexible, adaptable and, accordingly, stress-resistant, while the labour market demands are changing almost daily [2]. Not of less importance is the global socio-economic crisis that has engulfed the whole world in recent years. Therefore, most people have no confidence in the day 
to come, being in a state of constant tension and chronic stress, depression, anxiety, dependence on alcohol or psychoactive drugs [3]. In other words, the issue addresses an emotional burnout syndrome (EBS), that is, a response of a human's organism to a long-term effect of professional and other stress factors of medium intensity [4]. The WHO defines burnout as a result of "chronic stress at a working place that couldn't be dealt with" [5]. Disorders related to EBS are professional burnout, professional defect, overfatigue syndrome, chronic fatigue syndrome, exhaustion syndrome, existential emptiness, akedia, the syndrome of poisoning with people, etc. The EBS is a consequence of gradual loss of physical, emotional, and cognitive energy, which results in mental exhaustion, personal detachment, dissatisfaction with the work done, physical fatigue, etc. $[6,7]$. By its nature, EBS is a mechanism of psychological protection, which arises in response to psychotraumatic factors and is manifested in the form of partial or complete emotion exclusion resulting from occupational stressors. At the beginning of its development, the mentioned condition has a positive influence since it allows using energy reserves of the organism efficiently. However, under its prolonged duration, the EBS negatively affects the relations with others, in daily and professional activities [6].

According to the International Classification of Diseases X-revision (ICD-X), EBS can be attributed to the chapter "Problems related to difficulties in coping with life complications and managing one's own life": Z73.0 - burnout. Undoubtedly, EBS is an occupational disease that most often develops in people-related professions like medical officers, educators, managers, interpreters, salers, speakers, etc. [8]. In particular, emotional burnout syndrome occurs most frequently among medical professionals as they have higher occupational requirements in modern society. Besides, their work is accompanied by an extremely high psychological and emotional load, physical fatigue, underestimation of the social significance of their profession, and low wages in many countries [2,9]. Compared to the entire working population, the prevalence of EBS among physicians is very high. In particular, according to official statistics only, about $54.0 \%$ of medics reported EBS in the United States in 2014, which is twice as much as the entire working population [10]. The prevalence of EBS among healthcare workers continues to rise, with $62.1 \%$ of physicians under 55 years reporting the problem in 2019 , and $49.4 \%$ of physicians over 55 years suffering from EBS [11]. The prevalence of this disease is the highest among medical practitioners of intensive care units and amounts to almost $70.0 \%$ [12]. In France, the prevalence of EBS among general practitioners is $34.6 \%$ and $50.1 \%$ among intensive care physicians [2]. Also, EBS is quite widespread among nursing staff. According to a recent metaanalysis, the global prevalence of EBS among nurses is $11.23 \%$ [13]. Nurses of intensive care units are most often affected by emotional burnout, amounting to almost $86.0 \%$ following recent studies [12].

The relevance of studying the EBS problem among medical professionals is due to its extremely negative impact both on the quality of medical care provided to an individual and the entire national healthcare system of a particular country. It has been proven that workers suffering from this syndrome have a high rate of absenteeism, medical errors, and negative clinical consequences [14]. Noteworthy is also the negative economic consequences of EBS in medical professionals: for example, in the United States, the annual cost of physician turnover and reduction of clinical hours due to EBS is almost $\$ 4.6$ billion. At the organizational level, such costs are $\$ 7,600$ per year per physician [11]. In the United States, the annual costs associated with the nurse turnover and reduced hours due to EBS are estimated to be between $\$ 7.87$ million and $\$ 8.45$ million [15].

The problem of EBS became especially acute in the conditions of the world medical crisis [16] caused by the COVID-19 pandemic, which affected 210 countries worldwide [17]. As of April 22, 2020, more than 2.6 million people had been infected with COVID-19, and 180,000 people died [18]. For many decades, pandemics were considered weighty stressors for a good reason as they cause crisis not only in the health care system, but also in the financial, economic, social, and political spheres, which often led to serious consequences on a global scale [16]. It is absolutely fair to say that the COVID-19 pandemic has caused a global humanitarian crisis, resulting in psychological trauma and suffering in hundreds of millions of people. Furthermore, people are afraid of further spread in every country [18]. It is quite understandable and obvious that healthcare workers have a tremendous burden under such conditions. Physical fatigue due to irregular working hours and heavy workload through a continuous and large flow of patients, constant psychological stress, feelings of powerlessness and insecurity, uncertainty, fear for their lives and the lives of their relatives are the main factors that make doctors, paramedics, nurses, and other health workers extremely sensitive to stress [19]. In Italy alone, more 
than 12,000 healthcare workers were infected with COVID-19 at the end of April 2020, and 228 doctors and 26 nurses died [20]. Today, there are a large number of studies examining COVID-19 pathogenesis, treatments, responses (both local and global), etc. [21-23], but research works on the impact of the current pandemic on the mental health of people, particularly medical professionals, are missing. The need to study the impact of the COVID-19 pandemic on the mental health of medical professionals cannot be overemphasized, since such studies will allow developing effective measures to prevent and improve the state of mental health in medical professionals, increase their productivity, etc.

The aim of the study was to establish the features of emotional burnout syndrome and its connection with typological characteristics of the personality in nurses who work with COVID-19 patients.

\section{Methods}

A total of 120 female nurses who worked with COVID-19 patients were surveyed, of whom 60 $(50.0 \%)$ worked in outpatient settings together with general practitioners and $60(50.0 \%)$ worked in the intensive care unit. The mean age of the study participants was $(40.86 \pm 3.20)$ years and the mean medical service duration amounted to $(13.64 \pm 1.73)$ years. For further comparison, only nurses with the EBS were selected: Group 1 consisted of 30 outpatient nurses (average age $(39.92 \pm 3.48)$ years, average medical work experience $-(13.25 \pm 1.55)$ years $)$, and Group 2 comprised 44 intensive care unit nurses (average age $(40.74 \pm 3.61)$ years, average medical work experience $-(12.90 \pm 1.62)$ years $)$.

Inclusion criteria for the study were age 25 to 55 years; work experience as a nurse of 5 years or more; duration of work with COVID-19 patients of 3 months or more since inclusion in the study; and a diagnosed emotional burnout syndrome.

Exclusion criteria were age less than 25 years and older than 55 years; acute somatic pathology; chronic somatic pathology in acute stage; nurse's reluctance to participate in the study.

The study was conducted in compliance with international norms and principles of biomedical ethics: "Rules of Ethical Principles for Scientific Medical Research Involving Human Subjects" approved by the Declaration of Helsinki (1964-2013), ICH GCP Principles (1996), International Ethical Guidelines for Biomedical Research Involving Human Subjects of the Council for International Organization of Med- ical Sciences, Council of Europe Convention on Human Rights and Biomedicine (as of 04.04.1997), the EU Council Directive No. 609 (as of 24.11.1986), the study protocol and informed consent form were reviewed and approved by the I.M. Sechenov First Moscow State Medical University (Sechenov University) (Moscow, Russian Federation), the Volgograd State Medical University (Volgograd, Russian Federation), and the Bioethics Commission of the Amur State University (Blagoveshchensk, Russian Federation).

Only nurses who signed an informed consent form for study participation were included. They were provided with detailed information on the subject matter and tasks of the study, expected results and potential risks, as well as information on their right to refuse to take part in the study at any stage, upon request. Complete confidentiality was maintained throughout the study.

In the course of the research, the following techniques were employed: clinical anamnestic methods, clinical psychopathological methods, an inquiry method, and a bibliosemantic method.

During the research, the questionnaire of emotional burnout diagnostics by Boyko V.V. was used to examine the level of "burnout" symptoms and establish quantitative indexes of EBS phases and integral EBS index. When interpreting the results according to this technique, the attention was paid to separate symptoms, estimating their intensity from 0 to 30 points (with the value from 0 to 9 points - the symptom is not formed, from 10 to 15 points - the symptom is being formed, from 16 and more points - the symptom is formed). Symptoms scored 20 or more points were the leading ones in different phases of EBS. Phases are scored from 0 to 120 points: a value of 0 to 36 points indicates that a particular phase of EBS has not formed, 37 to 60 points - the phase is being formed, 61 or more points - the phase has been formed.

The Maslach Burnout Inventory (MBI) questionnaire was used to determine the level of emotional burnout, which included 3 scales: emotional exhaustion, depersonalization, and reduction of professional achievements. Each answer was estimated from 0 to 6 points, interpretation was made as follows: for emotional burnout, the sum of points from 0 to 15 indicated a low level of burnout, from 16 to 24 - an average level, 25 and more - high level; for depersonalization, from 0 to 5 points - low level, from 6 to 10 - an average level, from 11 and more - high level; for professional (personal) achievements 37 and more 
points - low level, from 31 to 36 - an average level, from 30 and less - high level.

The Personality Diagnostic Questionnaire (PDQIV) by Hyler was applied to establish the type of personality. The questionnaire is composed of 99 questions to be answered in a "true-false" format. A total score of $<20$ implies no personality disorder, from 20 to 30 points means a further evaluation is required, and $>30$ scores indicates the presence of personality disorder.

Anxiety and depression were assessed using the Hospital Anxiety and Depression Scale (HADS), which consists of 2 parts (7 questions each): part 1 - assessment of the anxiety level and part $2-$ assessment of the depression level. A score from 0 to 7 indicated the absence of symptoms of anxiety and depression, scores from 8 to 10 points implied the presence of subclinical anxiety/depression, and scores from 11 points and above meant signs of clinical anxiety/depression.

Statistical data processing was performed by calculating the arithmetic mean and standard deviation. The significance level of $5 \%(p<0.05)$ was used in assessing statistical intergroup differences of certain parameters. Samples were checked for normality by the Shapiro-Wilkie method. Statistical significance of quantitative indices between two comparison groups was determined using Wilcoxon-Mann-Whitney Ucriterion. The correlation between the indices was assessed by the Spearman correlation coefficient. The frequency of symptoms and SEB phases was assessed using the odds ratio (OR) calculation technique. Mathematical data processing was performed using Statistica for Windows 10 Pro software (Stat Soft Inc., USA) and Microsoft Excel 2013 (Microsoft, USA).

\section{Results}

The study design is shown in Fig. 1. Comparison groups were comparable for age and duration of service. It has been established that EBS of varying severity was present in $30(50.0 \%)$ outpatient nurses and in $44(73.3 \%)$ nurses working in intensive care $(\mathrm{OR}=2.44,95 \%$ CI $[1.16-5.16], p<0.05)$.

When analysing the structure of EBS, the tension phase was found in 16 (53.3\%) nurses of the outpatient unit, the resistance phase in $8(26.7 \%)$, and the exhaustion phase in 6 (20.0\%). Among nurses of the intensive care unit, the tension phase was present in $7(15.9 \%)$, the resistance phase in 28 (63.6\%), and the exhaustion phase in $9(20.5 \%)$ individuals. When calculating the odds ratio of each phase among the study groups, a statistically significant difference was found between outpatient and intensive care nurses in the frequency of the tension $(\mathrm{OR}=6.04,95 \% \mathrm{CI}$ [2.05-17.79], $p<0.05)$ and resistance phases presence $(\mathrm{OR}=4.81,95 \%$ CI [1.74-13.29], $p<0.05)$; there was no statistically significant intergroup difference $(p>0.05)$ in the frequency of exhaustion phase presence between individuals in both comparison groups $(\mathrm{OR}=1.03,95 \% \mathrm{CI}$ [0.32-3.27], $p>0.05)$.

The evaluation of each EBS phase in points (Table 1) has showed that in outpatient nurses, the phase of tension has already been formed, and the phases of resistance and exhaustion are being formed. In intensive care nurses, the phase of resistance has been formed, and the phase of tension and exhaustion was in the formation stage.

Besides, it has been established that nurses in both groups had subclinical anxiety: outpatient nurses $(7.58 \pm 0.81)$ points, intensive care $-(9.04 \pm 0.93)$ points with no statistically significant intergroup difference $(p>0.05)$. Depression was absent in both groups under study: outpatient nurses had a $(5.31 \pm 0.47)$ score, intensive care $-(6.09 \pm 0.62)$ score with no statistically significant intergroup difference $(p>0.05)$.

When analysing the MBI questionnaire data, both groups of respondents were characterized by an average value of emotional burnout level: for outpatient nurses, it was $(18.48 \pm 1.25)$ scores, for intensive care nurses $-(24.57 \pm 1.46)$ scores with a statistically significant intergroup difference $(p<0.05)$. In outpatient nurses, the average value of depersonalization level was $(4.04 \pm 0.73)$ points, in intensive care nurses $(7.85 \pm 0.8)$ points respectively with the available statistically significant intergroup difference in the index $(p<0.05)$. The average value in the level of professional achievement reduction in outpatient nurses was $(45.37 \pm 4.61)$ points, in intensive care nurses $(40.96 \pm 3.68)$ points with no significant intergroup difference in the index $(p>0.05)$. At the same time, it was found that a high level of emotional exhaustion was present in 7 (23.3\%) outpatient nurses, an average level - in 14 (46.7\%), low - in 9 (30.0\%). Among intensive care nurses, $15(34.1 \%)$ had a high level of emotional exhaustion, $23(52.3 \%)$ had an average level, and only $6(13.6 \%)$ had a low level. Regarding depersonalization, outpatient nurses had a high level of this characteristic in $2(6.7 \%)$ people, an average level in $12(40.0 \%)$ people, and a low level in 16 $(53.3 \%)$ people. Among intensive care nurses, a high level of depersonalization was in 5 (11.4\%) people, medium - in $30(68.2 \%)$ people, low - in 9 peo- 


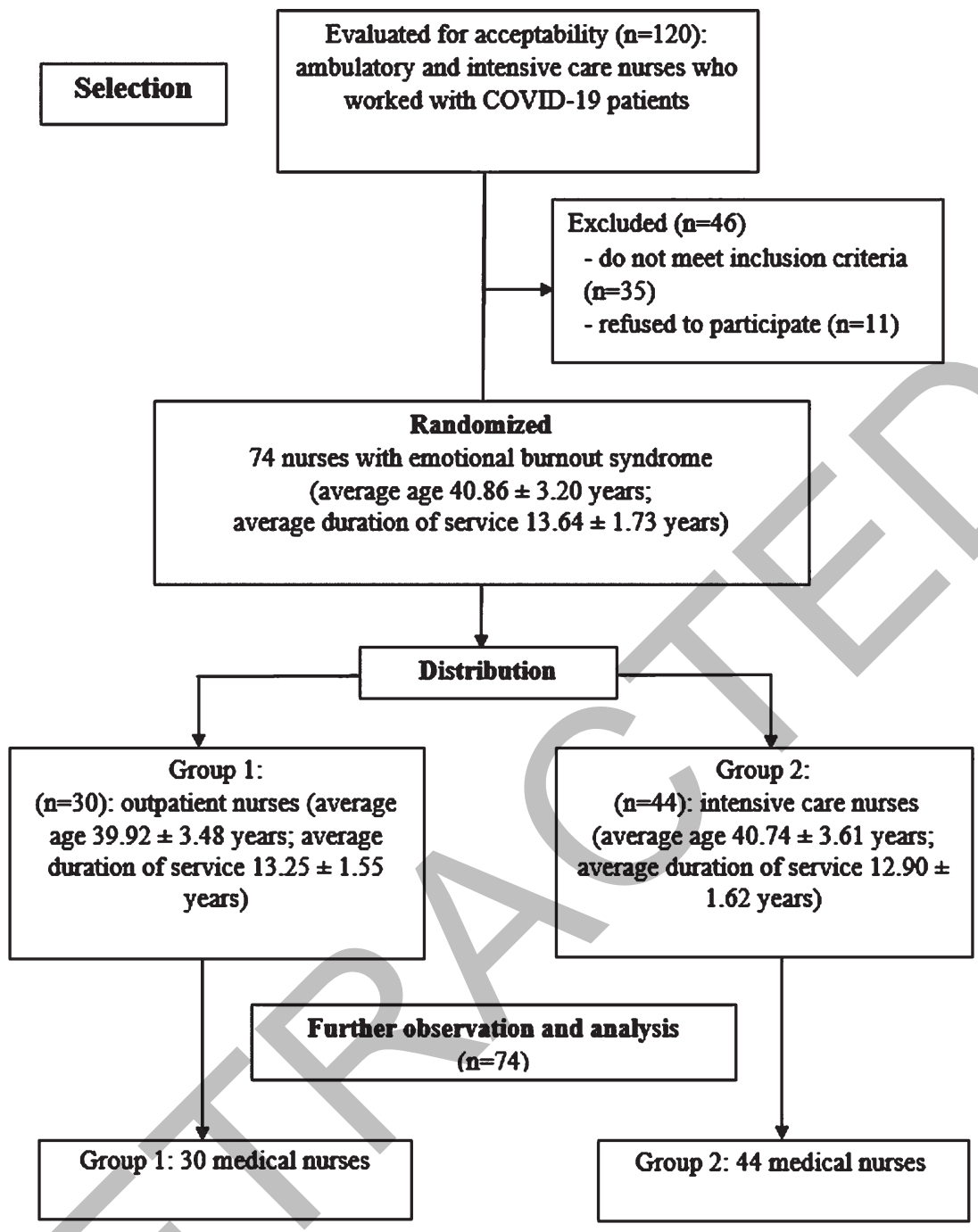

Fig. 1. Study design.

Table 1

Phases of emotional burnout in nurses working with COVID-19 patients, $M \pm \mathrm{m}$

\begin{tabular}{lcc}
\hline EBS phase & $\begin{array}{c}\text { Outpatient nurses } \\
(n=30)\end{array}$ & $\begin{array}{c}\text { Intensive care nurses } \\
(n=44)\end{array}$ \\
\hline Tension phase, points & $80.64 \pm 5.16$ & $57.38 \pm 4.83^{*}$ \\
Resistance phase, points & $58.71 \pm 5.34$ & $86.45 \pm 7.22^{*}$ \\
Exhaustion phase, points & $54.45 \pm 3.20$ & $59.60 \pm 4.59$ \\
\hline
\end{tabular}

${ }^{*}$ The difference is statistically significant compared to ambulatory care nurses $(p<0.05)$.

ple (20.4\%). There was no high level of professional achievement reduction among outpatient nurses, the average level was present in $10(33.3 \%)$ people, and the low level was present in $20(66.7 \%)$ people. A high level of reduction in professional success was found in $3(6.8 \%)$ intensive care nurses, an average level in $17(38.6 \%)$, and a low level in $24(54.6 \%)$.
Based on the performed correlation analysis, direct correlations between emotional exhaustion and length of service has been established as $(r=0.90$, $p<0.05)$, depersonalization as $(r=0.82, p<0.05)$, anxiety as $(r=-0.92, p<0.05)$, and an inverse correlation with personality reduction as $(r=-0.85$, $p<0.05$ ). 


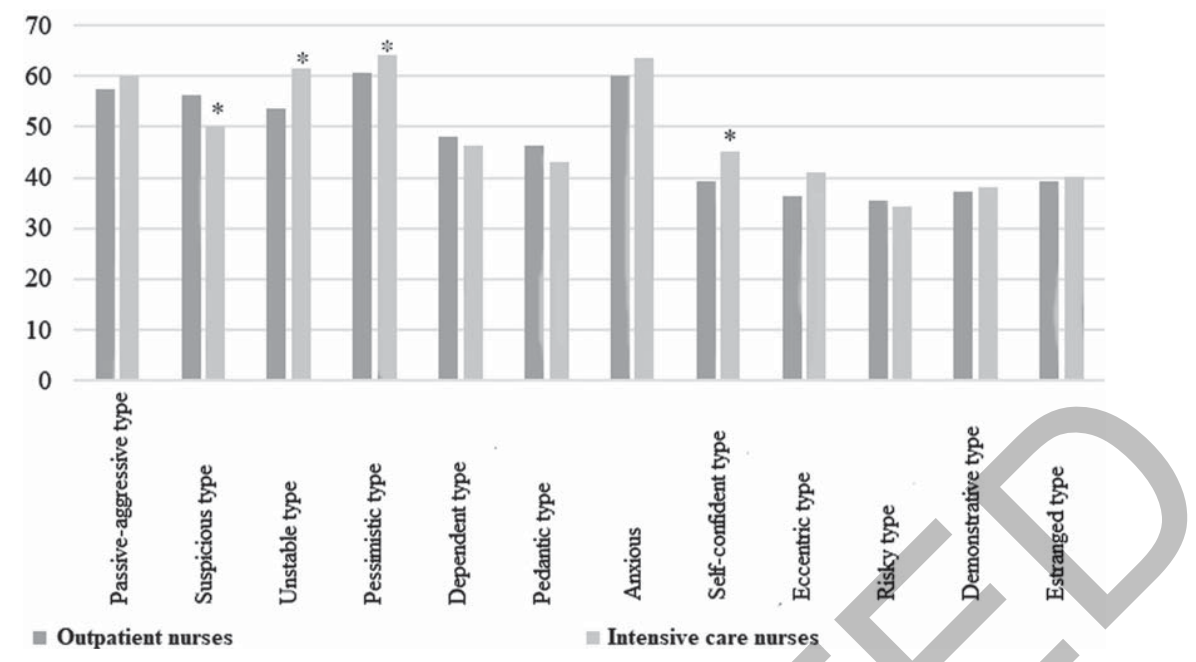

Fig. 2. Comparison of average PDQ-IV questionnaire values in outpatient and intensive care nurses. *The difference is statistically significant compared to ambulatory care nurses $(p<0.05)$.

While studying the personality disorders based on processing data from the PDQ-IV questionnaire, the typological profile of personality among outpatient and intensive care nurses was found to be generally the same (Fig. 2).

Both groups had obvious peaks in the following personality types: passive-aggressive, unstable, pessimistic, and anxious. There was a statistically significant inter-group difference $(p<0.05)$ between the average values of unstable, suspicious, pessimistic, and self-confident personality types.

Based on the correlation analysis, the presence of links between the level of emotional burnout and passive-aggressive $(r=0.46, p<0.5)$, suspicious $(r=0.41, p<0.05)$, unstable $(r=0.54, p<0.05)$, anxious $(r=0.59, p<0.05)$, and pessimistic $(r=0.40$, $p<0.05)$ personality types has been established.

\section{Discussion}

The research is devoted to studying the features of emotional burnout syndrome in outpatient and intensive care unit nurses, working directly with COVID-19 patients daily. This fact is undoubtedly an additional psychotraumatic factor and a trigger for the occurrence of EBS disorder among medical workers. It is worth specifying that nurses with at least 5 years of medical experience were included in the study since lack of work experience or little experience is also, in the author's opinion, an additional stress factor in conditions of coronavirus pandemic. The results of this study demonstrate a high prevalence of EBS among nurses in both groups, working with COVID-19 patients, namely, in $50.0 \%$ of outpatient nurses and $73.3 \%$ of intensive care nurses. Such a high incidence among nurses is not only because their activity is associated with daily communication with different people, but also due to the high responsibility for health and life of these people. The presence of a statistically significant difference in the prevalence of EBS between the studied groups $(\mathrm{OR}=2.44$, 95\% CI [1.16-5.16], $p<0.05)$ confirms that work in an intensive care unit is accompanied by a large action of various stressors, which caused a high prevalence of the specified syndrome among intensive care nurses. Some differences were found in the structure of the EBS phases: in particular, the tension phase was present in the largest proportion of outpatient nurses, namely, $53.3 \%$, which was significantly higher than in intensive care nurses, among whom this phase was observed in $15.9 \%$ of persons $(\mathrm{OR}=6.04,95 \% \mathrm{CI}$ [2.05-17.79], $p<0.05)$. However, among ICU nurses, the resistance phase was more frequently recorded, namely, in $63.3 \%$ of individuals, which was 2.39 times $(p<0.05)$ more frequent compared to ambulatory care nurses, among whom the specified phase was present in $26.7 \%$ of individuals $(\mathrm{OR}=4.81,95 \%$ CI [1.74-13.29], $p<0.05)$. Such differences in the structure of the EBS phases between the studied groups can probably be explained by the fact that intensive care nurses are used to working in more extreme and harsh conditions (daily patient deaths, severe health condition in patients, emotionality of relatives, a need for rapid response different situations, etc.) compared to outpatient nurses, whose 
work in normal conditions is calmer. For this reason, the resistance phase (resistance to stress, the desire to achieve psychological comfort, attempts to reduce the effects of stressors) is more common among ICU nurses. The tension phase, in turn, is the trigger for the EBS formation, its basis in outpatient nurses. This fact is confirmed by the results of evaluating EMS phases in scores (see Table 1): outpatient nurses are characterized by only tension phase $(80.64 \pm 5.16$ points), while intensive care nurses - by resistance phase ( $86.45 \pm 7.22$ points).

The presence of a higher level of emotional exhaustion in intensive care nurses $((24.57 \pm 1.46)$ points versus $(18.48 \pm 1.25)$ points in outpatient nurses $(p<0.05))$ indicate a greater degree of decrease in their general mood background, emotional lability, and mental exhaustion. A significantly higher level of depersonalization in ICU nurses $(7.85 \pm 0.80)$ points versus $(4.04 \pm 0.73)$ points in outpatient nurses $(p<0.05)$ may indicate their more emotional detachment, indifference, perhaps even formal performance of duties without empathy, which is related to the fact that over years of working in the ICU, patient death or suffering is a common occurrence for them. This is confirmed by the correlations established between emotional exhaustion and years of service $(r=0.90$, $p<0.05)$, depersonalization $(r=0.82, p<0.05)$.

The results of this research established that EBS leads to personality disorders, which is confirmed by the revealed correlation links between the level of emotional burnout and passive-aggressive $(r=0.46$, $p<0.05)$ ), suspicious $(r=0.41, p<0.05)$, unstable $(r=0.54, p<0.05)$, anxious $(r=0.59, p<0.05)$, and pessimistic $(r=0.40, p<0.05)$ personality types. The correlations established suggest that nurses with "anxious" and "unstable" personality types are most susceptible to emotional exhaustion syndrome.

The results obtained are generally consistent with those of other studies conducted in this sphere [19, 20, 24]. In particular, the study conducted in China found that behind the EBS subscales, exhaustion was present in $78.5 \%$ of nurses and depersonalization in $92.5 \%$. Nurses with long-term COVID-19 quarantine unit employment were characterized by significantly higher emotional exhaustion $(\mathrm{OR}=2.72,95 \%$ CI [0.02-5.42]; $p=0.049)$ and depersonalization $(\mathrm{OR}=1.14,95 \% \mathrm{CI}[0.10-2.19] p=0.033)$. In terms of age, greater emotional exhaustion $(\mathrm{OR}=2.96,95 \%$ CI [0.11-5.82]; $p=0.042)$ and less personal achievement $(\mathrm{OR}=3.80,95 \%$ CI [0.47-7.13]; $p=0.033)$ [24] was more typical for younger nurses compared to older colleagues. When comparing the cited study with the results of this study, the prevalence of EBS was established to be lower among nurses of this country. Probably, it can be explained by the fact that Chinese healthcare workers were the first to encounter the COVID-19 pandemic, when no treatment regimens, prevention measures, consequences were unknown, which was a weighty psychotraumatic factor.

In another study that involved all medical workers (both doctors and nursing staff), working with COVID-19 patients, $35.7 \%$ of medical workers have been established to have moderate emotional burnout and $31.9 \%$ - severe form. Moderate depersonalization was observed in $14.0 \%$, and severe - in $12.1 \%$ of individuals. Moderate level of decrease in professional achievements was recorded in $40.1 \%$, and severe form -in $34.3 \%$, with female gender and nursing work being predictors of EBS [20].

Afshari et al. [25] provided interesting evidence while studying the resistance rate and its predictors in nurses who worked in hospitals with COVID-19 patients. Nurses' resistance was shown to be quite low (mean resistance score was $61.18 \pm 14.8$ for CDRISC), while education and work experience have been identified as factors that enhance resistance to stress and tension.

\section{Conclusions}

The work of nurses under conditions of the COVID-19 pandemic is characterized by the high prevalence of emotional burnout syndrome, which is a consequence of mental adaptation disorder to professional stressors. This study revealed that burnout syndrome of varying severity was present in $50.0 \%$ of outpatient nurses and $73.3 \%$ of intensive care nurses $(\mathrm{OR}=2.44,95 \%$ CI $[1.16-5.16] ; p<0.05)$. In the dynamic structure of emotional burnout syndrome in outpatient nurses, the tension phase prevailed in $53.3 \%$ of individuals versus $15.9 \%$ of intensive care nurses $(\mathrm{OR}=6.04,95 \%$ CI [2.05-17.79], $p<0.05)$. In ICU nurses, the resistance phase was predominant in $63.3 \%$ of individuals versus $26.7 \%$ of outpatient nurses $(\mathrm{OR}=4.81,95 \% \mathrm{CI}$ [1.74-13 29]; $p<0.05)$ ), who was in the formed phase. Intensive care nurses were characterized by higher levels of emotional burnout $((24.57 \pm 1.46)$ versus $(18.48 \pm 1.25)$ scores $(p<0.05))$ and depersonalization $((7.85 \pm 0.80)$ versus $(4.04 \pm 0.73)$ scores $(p<0.05))$ compared to ambulatory nurses. Among personal factors of emotional burnout syndrome a number of typological 
features with signs of passive-aggressive, suspicious, unstable, anxious, and pessimistic personality types was established. The performed research allows assuming the interrelations between emotional burnout and typological features of personality in nurses. The received results enable the development of effective measures for prevention and correction of emotional burnout syndrome in nurses working in intense COVID-19 pandemic conditions, considering typological features of a particular personality.

\section{Limitations}

It would be useful to compare the personality characteristics and typology of nurses working with COVID-19 patients and nurses who have no such experience. In that way, it would be clearer how the characteristics of working in a pandemic environment related to COVID-19 affects EBS outbreaks in nurses. However, this is extremely difficult to do under real-life conditions, since each nurse has some connection to COVID-19 patients. It is thus not possible to exclude the influence of this factor up to now. In the future, a retrospective analysis of the emotional burnout syndrome features and its influence on personality typology in nurses before COVID-19 pandemic is planned. An option to undertake a similar study following the end of the COVID-19 pandemic is also being discussed.

\section{Conflict of interest}

The authors declare that they have no competing interests.

\section{Funding}

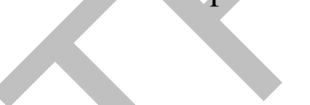

This research did not receive any specific grant from funding agencies in the public, commercial, or not-for-profit sectors.

\section{References}

[1] Chirico F, Magnavita N. Burnout syndrome and metaanalyses: need for evidence-based research in occupational health. Comments on prevalence of burnout in medical and surgical residents: a meta-analysis. Int J Environ Res Public Health. 2020;17(3):741. https://doi.org/10.3390/ ijerph17030741.
[2] Moukarzel A, Michelet P, Durand AC, Sebbane M, Bourgeois S, Markarian T, Bompard C, Gentile S. Burnout syndrome among emergency department staff: prevalence and associated factors. BioMed Res Int. 2019. https://doi.org/10.1155/2019/6462472.

[3] Vlachou EM, Damigos D, Lyrakos G, Chanopoulos K, Kosmidis G, Karavis M. The relationship between burnout syndrome and emotional intelligence in healthcare professionals. Health Sci J. 2016;10(5):2. https://doi.org/10.4172/1791-809X.1000100502.

[4] Friganovic A, Selic P, Ilic B, Sedic B. Stress and burnout syndrome and their associations with coping and job satisfaction in critical care nurses: a literature review. Psychiatria Danubina. 2019;31(1):21-31.

[5] Chatterjee R, Wroth C. WHO redefines burnout as a "syndrome" linked to chronic stress at work; 2019 [accessed January 27, 2020]. Available from: https://www.npr.org/sec tions/health-shots/2019/05/28/727637944/who-redefinesburnout-as-a-syndrome-linked-to-chronic-stress-at-work.

[6] Golonka K, Mojsa-Kaja J, Popiel K, Marek T, Gawlowska M. Neurophysiological markers of emotion processing in burnout syndrome. Frontiers Psychol. 2017;8:2155. https://doi.org/10.3389/fpsyg.2017.02155.

[7] Johnson SS. The Editor's desk: burnout. Am J Health Prom. 2020;34(5):563-575. https://doi.org/10.1177/08901171209 20488a.

[8] World Health Organization. Burn-out an "occupational phenomenon": International Classification of Diseases; 2019 [accessed January 27, 2020]. Available from: https://www.who.int/news/item/28-05-2019-burn-out-anoccupational-phenomenon-international-classification-ofdiseases.

[9] Bakhamis L, Paul DP, Smith H, Coustasse A. Still an epidemic: the burnout syndrome in hospital registered nurses. Health Care Manag. 2019;38(1):3-10. https://doi.org/10.10 97/hcm.0000000000000243.

[10] Shanafelt TD, Hasan O, Dyrbye LN, Sinsky C, Satele D, Sloan J, West CP. Changes in burnout and satisfaction with work-life balance in physicians and the general US working population between 2011 and 2014. Mayo Clin Proc. 2015;90(12):1600-1613. https://doi.org/10.1016/ j.mayocp.2015.08.023.

[11] Han S, Shanafelt TD, Sinsky CA, Awad KM, Dyrbye LN, Fiscus LC, Trockel M, Goh J. Estimating the attributable cost of physician burnout in the United States. Ann Intern Med. 2019;170(11):784-790. https://doi.org/10.7326/m181422.

[12] Costa DK, Moss M. The cost of caring: emotion, burnout, and psychological distress in critical care clinicians. Ann Am Thoracic Soc. 2018;15(7):787-790. https://doi.org/ 10.1513/annalsats.201804-269ps.

[13] Woo T, Ho R, Tang A, Tam W. Global prevalence of burnout symptoms among nurses: A systematic review and meta-analysis. J Psych Res. 2020;123:9-20. https://doi.org/ 10.1016/j.jpsychires.2019.12.015.

[14] Dyrbye LN, Awad KM, Fiscus LC, Sinsky CA, Shanafelt TD. Estimating the attributable cost of physician burnout in the United States. Annals Intern Med. 2019;171(8):600601. https://doi.org/10.7326/119-0522.

[15] Hetzel-Riggin MD, Swords BA, Tuang HL, Deck Joshua M, Spurgeon NS. Work Engagement and Resiliency Impact the Relationship Between Nursing Stress and Burnout. Psychol. Rep. 2019;123(5):1835-1853.

[16] Sahni S, Kumari S, Pachaury P. Building emotional resilience with big five personality model against COVID-19 
<smiles>C1=C[C@H]2C=C[C@@H]1C2</smiles> 